\title{
Hidden Stories: Topic Modeling in Hydrology Literature
}

\author{
Mashrekur Rahman $^{1}$, Jonathan M. Frame ${ }^{1}$, Jimmy Lin ${ }^{2}$, Grey S. Nearing ${ }^{1,3}$ \\ ${ }^{1}$ Department of Geological Sciences, University of Alabama \\ ${ }^{2}$ David R. Cheriton School of Computer Science, University of Waterloo \\ ${ }^{3}$ Upstream Tech Public Benefit Company, Natel Energy
}

\section{Key Points:}

- Topic Modeling is a form of unsupervised machine learning for Natural Language Processing (NLP)

- Topic Modeling can provide a high-level overview of topics and trends in hydrology literature

- This is a first step toward building a tool to help researchers navigate and synthesize a growing body of literature

\section{Keywords:}

- Hydrology Literature

- Science Communication

- Machine Learning

- Unsupervised Learning

- Natural Language Processing

- Topic Modeling 


\begin{abstract}
Hydrologic research generates large volumes of peer-reviewed literature across a number of evolving sub-topics. It's becoming increasingly difficult for scientists and practitioners to synthesize this full body of literature. This study explores topic modeling with Latent Dirichlet Allocation (LDA) as a form of unsupervised learning applied to 42,154 article-abstracts from six high-impact (Impact Factor $>0.9$ ) journals (Water Resources Research WRR, Hydrology and Earth System Sciences HESS, Journal of Hydrology $J H$, Hydrological Processes HP, Hydrological Sciences Journal HSJ, Journal of Hydrometeorology $J H M$ ) to provide a high-level contextual analyses of hydrologic science literature since 1991. We used a hybrid quantitative/qualitative approach to label a number of broad topics in this body of literature, and used these labeled topics to analyze topic trends, inter-topic relationships, and journal diversity. As an example of what we can learn from this type of analysis, results showed that data-driven research topics are gaining in popularity while some subsurface related topics lose popularity within our journal set and time period. While no journal in our sample was completely homogeneous, JHM and WRR exhibited the most notable preferences for certain topics over others. The methods and outcomes of this paper are potentially beneficial to scientists and researchers who aim to gain a contextual understanding of the existing state of hydrologic science literature. In the long term, we see topic modeling as a tool to help increase the efficiency of literature reviews, science communication, and science-informed policy and decision making.
\end{abstract}

